\title{
LA RELEVANCIA DE LOS PROGRAMAS EDUCATIVOS Y FORMATIVOS COMO ELEMENTOS FAVORECEDORES DEL PROCESO DE REINSERCIÓN SOCIAL Y LABORAL DE LOS MENORES INFRACTORES
}

\author{
Juan Miguel Fernández-Campoy \\ José Manuel Aguilar-Parra \\ Juana Celia Domínguez \\ Pablo Rueda-Rosas \\ Ma Carmen Lozano Segura \\ Universidad de Almería. jfc105@ual.es \\ https://doi.org/10.17060/ijodaep.2017.n1.v2.953
}

Fecha de Recepción: 22 Febrero 2017

Fecha de Admisión: 1 Abril 2017

\section{RESUMEN.}

Las sociedades del momento suelen recurrir, en un claro intento de organizar, gestionar y facilitar el desarrollo del proceso de reinserción social y laboral de los menores infractores, al empleo de un amplio elenco de programas y estrategias con las que intentan prevenir la aparición de las conductas delictivas e ilícitas 0 , en el caso de que éstas se acaben desarrollando, intervenir para reconducirlas hacia otro tipo de conductas sociales y laborales que acaben siendo ampliamente aceptadas y valoradas por los principales entramados sociales y laborales. Entre estos programas y estrategias de prevención e intervención que se vienen desarrollando e implementando, en los últimos años la comunidad científica comienza a aceptar y a reconocer los enormes beneficios derivados de trabajar con estos menores programas de naturaleza educativa y formativa, siendo los más significativos la adquisición de un amplio abanico de valores y actitudes personales, sociales y laborales (empatía, solidaridad, tolerancia, cooperación, sacrificio, autoestima, autocontrol, etc.) que acaban facilitando su plena y efectiva integración en las estructuras sociales, comunitarias y laborales. Para realizar buenas investigaciones dentro de este ámbito, por tanto, resulta imprescindible determinar todas aquellas variables y factores que se encuentran presentes en este tipo de programas educativos y formativos. Por lo tanto, en el presente estudio se analizan los principales trabajos desarrollados sobre la temática objeto de estudio, con la intención de analizar y describir los distintos factores y variables que están presentes en los programas educativos y formativos, así como los más destacados beneficios y parabienes que pueden reportar a los menores infractores que se acaben convirtiendo en usuarios habituales de los mismos. De esta manera, es posible delimitar el punto desde el que habrán de partir las sucesivas investigaciones empíricas y programas que traten de gestionar el proceso de reinserción social y laboral de los menores infractores. 


\section{LA RELEVANCIA DE LOS PROGRAMAS EDUCATIVOS Y FORMATIVOS COMO ELEMENTOS FAVORECEDORES DEL PROCESO DE REINSERCIÓN SOCIAL Y LABORAL DE LOS MENORES INFRACTORES}

Palabras clave: Menores infractores, programas educativos y formativos, reinserción social y laboral, entorno socio-comunitario y laboral, educación, revisión.

\section{ABSTRACT.}

The relevance of educational and training programs as elements that favor the process of social and labor reintegration of juvenile offenders.

In a clear attempt to organize, manage and facilitate the process of social and labor reintegration of juvenile offenders, the societies of the moment tend to use a wide range of programs and strategies with which they try to prevent the emergence of Criminal and illicit behaviors or, in the event that they are developed, intervene to redirect them to other types of social and labor behavior that end up being widely accepted and valued by the main social and labor networks. Among these programs and strategies of prevention and intervention that have been developed and implemented, in recent years the scientific community begins to accept and recognize the enormous benefits derived from working with these smaller programs of an educational and formative nature, the most significant being the acquisition (Empathy, solidarity, tolerance, cooperation, sacrifice, self-esteem, self-control, etc.) that end up facilitating their full and effective integration into social, community and labor structures. In order to carry out good research in this field, therefore, it is essential to determine all those variables and factors that are present in this type of educational and training programs. Therefore, the present study analyzes the main works developed on the subject of study, with the intention of analyzing and describing the different factors and variables that are present in the educational and training programs, as well as the most outstanding benefits and Parabienes that can report to juvenile offenders who end up becoming habitual users of them. In this way, it is possible to delimit the point from which the successive empirical investigations and programs that try to manage the process of social reintegration and labor of the juvenile offenders will have to start.

Key words: Minor offenders, educational and training programs, social and labor reinsertion, socio-community and labor environment, education, revision.

\section{ANTECEDENTES DE LA TEMÁTICA.}

De un tiempo a esta parte, las sociedades de los países desarrollados vienen asistiendo, con gran preocupación y desazón, a un importante repunte de las actividades delictivas e ilícitas, que tienen como principales protagonistas a individuos que se encuentran en las etapas evolutivas de la infancia y la adolescencia, una situación que amenaza con dinamitar las principales estructuras sociales y laborales de dichos entramados socio-comunitarios, máxime si se tiene en cuenta que éstos tienen encomendada la responsabilidad, en un intento de asegurar su adecuada estructuración y funcionamiento, de garantizar, de manera efectiva y plena, el que todos estos menores infractores sean conveniente formados y reeducados, a fin de que, tras saldar la totalidad de las deudas que en su momento contrajeron con los sistemas y las estructuras judiciales, puedan reintegrarse en sus entramados sociales y laborales como ciudadanos activos y de pleno derecho, de manera que logren disponer de los elementos y recursos metodológicos y procedimentales que les posibiliten participar, de forma adecuada, en los mismos, contribuyendo a su correcto desarrollo, crecimiento y mejora (Burt, Resnick y Novick, 1998; Fernández-Campoy, Aguilar-Parra y Álvarez, 2013; Giller, Haggel y Rutter, 2000).

La reinserción social y laboral de los menores infractores, dada su magnitud y complejidad, se presenta como un exigente proceso que es conveniente planificar, de forma exhaustiva, para que logre reconducir la vida social, comunitaria y laboral de los menores delincuentes, una vez que éstos consigan saldar la totalidad de las deudas que contrajeron con las instituciones judiciales. Este 
hecho ha ocasionado, en los últimos años, frecuentes quebraderos de cabeza a los principales responsables de las diferentes administraciones que vienen asumiendo competencias en esta materia, además de diferentes formas de entender y de gestionar los aspectos más significativos de dicho proceso, dando lugar al desarrollo y a la implementación de un amplio abanico de estrategias y de programas de prevención e intervención, con orientaciones metodológicas y procedimentales bastante dispares entre sí, aunque con ciertos puntos comunes, sobre todo a la hora de destacar la conveniencia y la necesidad de hacer hincapié en el desarrollo, en el fortalecimiento y en la consolidación de los aspectos educativos y formativos de dicho colectivo de menores (De la Rosa, 2003; Farrington, 2000; Fernández-Campoy, 2008).

Lo que en la actualidad muy pocos investigadores se atreven a poner en entredicho es el hecho de que la educación se ha convertido en un importante instrumento que suele ocupar un lugar destacado en la mayoría de los programas y estrategias formativas de reinserción social y laboral de menores infractores que se vienen desarrollando e implementando en el presente porque, como muy bien se han encargado de demostrar los numerosos estudios empíricos que, sobre la materia, se han desarrollado a lo largo de las últimas décadas, lo que se persigue, en la gran mayoría de ellos, es tratar de formar a los menores delincuentes para que logren erradicar de su repertorio conductual todos aquellos comportamientos que les han llevado a distorsionar la calidad y la efectividad de sus relaciones e interacciones sociales y laborales, hasta el punto de acabar desarrollando comportamientos sociales y laborales inadecuados que, con relativa frecuencia, acaban siendo rechazados 0 cuestionados por las estructuras de los más destacados entramados sociales, comunitarios y laborales de los países desarrollados (Fernández-Campoy, Aguilar-Parra, Álvarez, Pérez-Gallardo y Salguero, 2013; Fernández-Campoy, Aguilar-Parra y Alías-García, 2012; Pérez, Amador y Vargas, 2011).

De acuerdo con los anteriores planteamientos científicos y con los postulados defendidos por investigadores como Babbis y Gangwisch (2010) y Coy y Torrente (1997), resulta conveniente destacar que son muchas las instituciones, organismos internacionales, gobiernos estatales y asociaciones sociales y civiles que comienzan a reconocer el importante valor de los programas educativos y formativos como destacados agentes de reinserción social y laboral de menores infractores, al lograr facilitar y fortalecer los principales mecanismos de cohesión social, comunitaria y laboral y reducir y corregir las desigualdades sociales y laborales, consiguiendo, de esta manera, crear el escenario adecuado para luchar contra la exclusión social y laboral de los colectivos más deprimidos y desfavorecidos.

A grandes rasgos, y como bien corroboran Alpízar, Calvo, Garita, Méndez, Mora, Loíra y Varela (2010), es posible afirmar que la actividad educativa y formativa se puede llegar a convertir en un importante instrumento, no sólo para desarrollar y mejorar diversos aspectos cognitivos e intelectuales en los individuos, sino también para consolidar en sus usuarios habituales importantes valores éticos y morales que les van a facilitar, en gran medida, su integración social y laboral, así como el establecimiento de relaciones e interacciones sociales y laborales mucho más ricas, sólidas y eficientes. Entre dichos valores es posible destacar los siguientes:

*Empatía.

*Solidaridad.

*Tolerancia.

*Cooperación.

*Sacrificio.

*Autoestima. 
${ }^{*}$ Autocontrol.

* Libertad.

${ }^{*}$ Paz.

Junto a las anteriores potencialidades como elementos configuradores de un amplio elenco de valores y de conductas ampliamente aceptadas por los entramados sociales, comunitarios y laborales, que van a facilitar a sus practicantes su rápida y efectiva integración social y laboral, también, y siempre y cuando se implementen de manera adecuada y efectiva, los programas educativos y formativos van a ser capaces de actuar como elementos inhibidores para el desarrollo de valores y de pautas de conducta que, al ser social y laboralmente reprobadas y desprestigiadas, pueden acabar obstaculizando sus posibilidades de desarrollo e integración en el seno de los entramados sociales, comunitarios y laborales, destacando entre ellas, en opinión de Shaw (2001) y de Castaño (2006), las siguientes:

*Violencia.

*Manipulación.

${ }^{*}$ Consumismo.

${ }^{*}$ Hedonismo.

*Triunfalismo.

*Utilitarismo.

En definitiva, y sobre la base de los datos y argumentaciones trabajadas hasta el momento, se podría llegar a la conclusión de que los programas educativos y formativos no solamente emergen como un extraordinario recurso educativo y formativo que favorece el desarrollo de valores social y laboralmente aceptados y que limita la aparición de otro tipo de valores que gozan de un gran desprestigio social y laboral, sino que también configuran un escenario propiciatorio para el desarrollo de destacados aspectos intelectuales, cognitivos y actitudinales que van a proporcionar a sus usuarios los recursos metodológicos y procedimentales necesarios para que se puedan llegar a convertirse, en un futuro no muy lejano, en ciudadanos activos y de pleno derecho que, con su buen hacer, puedan contribuir al crecimiento, a la transformación y a la mejora de los principales entramados y estructuras sociales, comunitarias y laborales (Ortega y Del Rey, 2006).

\section{OBJETIVOS DE LA INVESTIGACIÓN.}

Descubrir aquellos elementos y factores que el conocimiento científico suele atribuir a los programas educativos y formativos, en relación a su capacidad como vehículos de organización, de gestión y de desarrollo del proceso de reinserción social y laboral de los menores infractores.

Analizar aquellos elementos y factores que suelen estar presentes en los más destacados programas educativos y formativos que en la actualidad se vienen diseñando e implementando para tratar de prevenir e intervenir ante el desarrollo de las principales conductas delictivas e ilícitas que pueden llegar a acontecer durante las etapas evolutivas de la infancia y la adolescencia.

\section{MÉTODO.}

\section{Muestra.}

Para configurar la muestra que en el estudio se encargaría de la producción de los datos, se procedió a la realización de una completa y exhaustiva revisión de la literatura científica que, a lo largo del devenir histórico, se ha venido especializando en el estudio, análisis y descripción de las princi- 
pales potencialidades de los programas educativos y formativos, en lo que a la prevención y a la reducción de las conductas delictivas e ilícitas que suelen manifestar los menores infractores durante la etapas evolutivas de la infancia y la adolescencia se refiere.

\section{Instrumentos.}

Para recopilar los datos se han analizado las publicaciones científicas especializadas en la temática que se aborda en el estudio, publicaciones proporcionadas por las bibliotecas científicas y la red telemática de internet.

\section{Procedimiento.}

En primer lugar, se visitaron varias bibliotecas científicas y se consultaron los documentos técnicos presentes en internet, con la idea de conseguir determinar la forma en la que los programas y estrategias educativas y formativas pueden contribuir a prevenir y a reducir los efectos adversos derivados de las conductas delictivas e ilícitas que pueden llegar a poner en juego los menores infractores durante las etapas evolutivas de la infancia y la adolescencia, así como la manera en la que su implementación puede ayudar a enriquecer y a mejorar la calidad y la eficiencia de los procesos de reinserción social y laboral que se suelen seguir con dichos menores y, en última instancia, las principales características y señas de identidad de los más destacados programas educativos y formativos que en la actualidad se vienen desarrollando e implementando para que los menores infractores adquieran un amplio abanico de recursos metodológicos y procedimentales que les permitan desarrollar, implementar y adoptar eficientes rutinas y conductas sociales, comunitarias y laborales que les ayuden a incrementar sus posibilidades de reinserción social y laboral, toda vez que logren saldar las deudas que un día llegaron a contraer con las estructuras judiciales.

En una segunda fase, se analizaron los datos recopilados durante la fase de implementación del estudio.

Por último, se ha elaborado un informe con los resultados y conclusiones más significativas del estudio.

\section{Análisis de datos.}

El enfoque metodológico empleado en el estudio ha sido de corte cualitativo con instrumentos descriptivos, como son los estudios científicos que han tratado de analizar la manera en la que los programas y estrategias educativas y formativas pueden contribuir a prevenir y a reducir las consecuencias adversas derivadas de las conductas delictivas e ilícitas que pueden acabar exhibiendo los menores infractores durante las etapas evolutivas de la infancia y la adolescencia, así como la forma en la que su implementación puede llegar a favorecer un incremento en los niveles de calidad y de eficiencia de los procesos de reinserción social y laboral que se suelen aplicar a dichos menores y las principales características y señas de identidad de los más destacados programas educativos y formativos que en la actualidad se vienen desarrollando e implementando para que los menores infractores logren adquirir un amplio abanico de recursos metodológicos y procedimentales que les permitan desarrollar, implementar y adoptar eficientes rutinas y conductas sociales, comunitarias y laborales que les ayuden a incrementar sus posibilidades de reinserción social y laboral, toda vez que logren saldar la totalidad de sus deudas judiciales, todo ello con la intención de conseguir una interpretación significativa y contextualizada de los datos recabados en el estudio (Buendía, 1999).

\section{RESULTADOS.}

Uno de los principales quebraderos de cabeza a los que se vienen enfrentando las sociedades contemporáneas de los países desarrollados es el significativo incremento de las actividades delic- 


\section{LA RELEVANCIA DE LOS PROGRAMAS EDUCATIVOS Y FORMATIVOS COMO ELEMENTOS FAVORECEDORES DEL PROCESO DE REINSERCIÓN SOCIAL Y LABORAL DE LOS MENORES INFRACTORES}

tivas e ilícitas que tienen como destacados protagonistas a individuos que se encuentran inmersos en los periodos evolutivos de la infancia y la adolescencia, unas situaciones que, de no intervenir con gran celeridad y eficiencia, amenazan con hacer tambalear los cimientos y las principales estructuras sociales y laborales de dichos entramados sociales, comunitarios y laborales. De manera subsidiaria a esta problemática, que ya de por sí es difícil de contrarrestar, también resulta conveniente recordar que dichas sociedades tienen la responsabilidad, en un claro intento de asegurar su adecuado funcionamiento, de garantizar, de manera efectiva y plena, el que todos estos menores infractores sean conveniente formados y reeducados para que, toda vez que consigan saldar la totalidad de sus deudas judiciales, puedan llegar a reintegrarse en sus propios entramados sociales y laborales como ciudadanos activos y de pleno derecho, a fin de que consigan disponer de los elementos y recursos metodológicos y procedimentales que les posibiliten participar convenientemente en los mismos y, por tanto, puedan contribuir a su correcto desarrollo, crecimiento y mejora (Ghiso, 2012; Howell y Hawkins, 1998).

La reinserción social y laboral de los menores infractores, al configurarse como un proceso muy ambicioso y complejo, y para resultar verdaderamente efectivo, va a requerir de un exhaustivo mecanismo de planificación, de coordinación y de estructuración, con la idea de que pueda conseguir la importante tarea de reconducir la vida social, comunitaria y laboral de los menores delincuentes, toda vez que éstos consigan saldar la totalidad de las deudas contraídas con las principales administraciones judiciales. Esta ingente responsabilidad ha venido generando, en los últimos tiempos, enormes procesos de análisis y de toma de decisiones a los principales responsables de las diferentes administraciones que tienen asignadas competencias en esta materia, así como distintas formas de entender y de gestionar los aspectos más destacados de dicho proceso, lo que, a su vez, ha dado lugar al desarrollo y a la implementación de un amplio abanico de estrategias y de programas de prevención e intervención, con orientaciones metodológicas y procedimentales bastante dispares entre sí, aunque con ciertos puntos de coincidencia, los cuales se manifiestan, principalmente, a la hora de destacar la conveniencia y la necesidad de centrar la atención en el desarrollo, en el fortalecimiento y en la consolidación de los aspectos educativos y formativos de dicho colectivo de menores (González y Santiuste, 2004).

Lo que parece claro, a tenor de las principales señas de identidad de los elementos constitutivos del proceso de reinserción social y laboral de los menores infractores, es que la educación emerge como un importante y destacado componente que acaba jugando un trascendental papel en la mayoría de los programas y estrategias de reinserción social y laboral de menores infractores que se vienen desarrollando e implementando en la actualidad porque, si se hace caso de los datos emanados de las numerosas investigaciones científicas que, sobre la materia, se han desarrollado a lo largo de las últimas décadas, lo que se persigue, en la casi totalidad de los mismos, es formar a los menores delincuentes para que logren erradicar de su repertorio conductual todas aquellas pautas de comportamiento que han contribuido a distorsionar la calidad y la efectividad de sus relaciones e interacciones sociales y laborales, como la mejor estrategia para conseguir articular comportamientos sociales y laborales ampliamente aceptados, desde un punto de vista social, comunitario y laboral, que les facilite su rápido y efectivo proceso de integración en las principales estructuras de los más destacados entramados sociales, comunitarios y laborales de los países desarrollados (Fernández-Campoy, Aguilar-Parra, Lorenzo-Torrecillas y Alcaraz-Ibáñez, 2016).

Actualmente, y tomando como principal referencia los principales planteamientos científicos y metodológicos descritos hasta el momento, han sido muchas las instituciones, organismos internacionales, gobiernos estatales y asociaciones sociales y civiles que han empezado a adoptar e implementar los programas educativos y formativos, al reconocer su importante valor como agen- 
tes de transformación y de reinserción social y laboral de menores infractores, dadas sus innumerables contribuciones para el fortalecimiento de los principales mecanismos de cohesión social y laboral, a la misma vez que logran atenuar y corregir, de manera sensible, las principales desigualdades sociales y laborales, con lo que aseguran la creación de las condiciones propiciatorias para luchar contra la exclusión social y laboral de los colectivos sociales y laborales más deprimidos y desfavorecidos (Fernández-Campoy, Aguilar-Parra, Lorenzo-Torrecillas y Salguero, 2016).

Por tanto, parece ampliamente constatado el hecho de que las actividades educativas y formativas, siempre y cuando se lleven a cabo con cierta continuidad en el tiempo y se participe de las mismas de forma adecuada y responsable, pueden llegar a configurarse como un destacado instrumento, no sólo para desarrollar y mejorar diversos aspectos cognitivos e intelectuales en los individuos, sino también para afianzar en sus usuarios habituales una serie de valores éticos y morales (empatía, solidaridad, tolerancia, cooperación, sacrificio, autoestima, autocontrol, libertad, paz, entre otros) que les va a facilitar, de manera considerable, el desarrollo y la gestión de su proceso de integración social, comunitaria y laboral, así como el establecimiento de relaciones e interacciones sociales y laborales mucho más ricas, sólidas y eficientes (Nieto, 2010).

Además de las potencialidades de los programas educativos y formativos como elementos generadores de valores y de conductas ampliamente reconocidas y aceptadas por las principales estructuras de los entramados sociales, comunitarios y laborales, que, sin duda, van a facilitar a sus usuarios una rápida y efectiva integración social y laboral, también es posible reconocer su importancia como elementos limitantes o inhibidores para el desarrollo de diversos valores y pautas conductuales (violencia, manipulación, consumismo, hedonismo, triunfalismo, utilitarismo, entre otros) que, al experimentar altos niveles de marginalidad y de desprestigio social y laboral, pueden acabar perjudicando los potenciales niveles de desarrollo y de integración social, comunitaria y laboral de los sujetos que los acaben llevando a la práctica (Blatier, 2002).

\section{DISCUSIÓN/CONCLUSIONES.}

Si hay algo que preocupa sobremanera a las sociedades actuales de los países desarrollados es el progresivo incremento de las actividades delictivas e ilícitas que tienen como principales protagonistas a individuos que se encuentran cursando los periodos evolutivos de la infancia y la adolescencia, unas situaciones que, de no intervenir con inmediatez y eficacia, amenazan con poner en jaque a sus principales estructuras sociales, comunitarias y laborales. Además de esta situación, que no resulta fácil de erradicar, no se puede obviar la circunstancia de que dichas sociedades tienen la responsabilidad, en un intento de asegurar su adecuada configuración, de garantizar, de manera real y efectiva, el que todos estos menores infractores sean conveniente formados y reeducados para que, toda vez que consigan saldar las deudas que contrajeron con los sistemas y las estructuras judiciales, puedan llegar a reintegrarse en sus propios entramados sociales, comunitarios y laborales como ciudadanos activos y de pleno derecho, a fin de que obtengan los elementos y recursos metodológicos y procedimentales que les posibiliten participar adecuadamente en los mismos y, por tanto, contribuir a su correcto desarrollo, crecimiento y mejora (Debarbieux y Blaya, 2006; Fernández-Campoy, Aguilar-Parra y López-Liria, 2015; Fernández-Campoy, Pérez-Gallardo, León y Salguero, 2014; Nieto, 2012; Schonert-Reichl, 2000).

El proceso de reinserción social y laboral de los menores infractores, dada su relevancia, surge como un complejo proceso que, para lograr llegar a buen término, va a requerir de un coherente mecanismo de planificación y de coordinación que ayude a reconducir la vida social, comunitaria y laboral de los menores delincuentes, toda vez que consigan saldar las deudas contraídas con las principales administraciones de justicia. Esta destacada responsabilidad ha venido generando, en 


\section{LA RELEVANCIA DE LOS PROGRAMAS EDUCATIVOS Y FORMATIVOS COMO ELEMENTOS FAVORECEDORES DEL PROCESO DE REINSERCIÓN SOCIAL Y LABORAL DE LOS MENORES INFRACTORES}

los últimos tiempos, frecuentes quebraderos de cabeza a los principales responsables de las diferentes administraciones con competencias en esta materia, así como distintas formas de entender y de gestionar los principales aspectos de dicho proceso, lo que, a su vez, ha dado lugar al desarrollo y a la implementación de un amplio abanico de estrategias y de programas de prevención e intervención, con orientaciones metodológicas y procedimentales bastante dispares entre sí, aunque con ciertos puntos coincidentes, los cuales se manifiestan, sobre todo, a la hora de destacar la conveniencia y la necesidad de centrar su intervención en el desarrollo, en el fortalecimiento y en la consolidación de los aspectos educativos y formativos de dicho colectivo de menores (FernándezCampoy, 2014; Krmpotic y Farré, 2008; Perea, Calvo y Anguiano, 2010; Sherman et Al, 1997).

Si se pone el acento en los elementos definitorios del escenario que se ha ido esbozando a lo largo y ancho del párrafo precedente, resulta inevitable considerar a la educación como un destacado componente que acaba jugando un trascendental papel en la mayoría de los programas y estrategias de reinserción social y laboral de menores infractores que se vienen desarrollando e implementando a día de hoy porque, si se hace caso de las conclusiones alcanzadas por las principales investigaciones científicas que, sobre la materia, se han desarrollado durante las últimas décadas, lo que se persigue, en la mayoría de los mismos, es formar a los menores delincuentes para que logren erradicar de su repertorio conductual todas aquellas pautas de comportamiento que les han ayudado a distorsionar la calidad y la efectividad de sus relaciones e interacciones sociales y laborales, como la mejor estrategia para conseguir configurar comportamientos sociales y laborales aceptados y adecuados, desde un punto de vista social, comunitario y laboral, que les facilite un efectivo proceso de integración en las principales estructuras de los más destacados entramados sociales, comunitarios y laborales de los países desarrollados (Estévez, Martínez, Moreno y Musitu, 2006; Martínez, Musitu, Amador y Monreal, 2012).

Sobre los cimientos que sustentan a los planteamientos científicos y metodológicos descritos hasta aquí, resulta conveniente precisar que son muchas las instituciones, organismos internacionales, gobiernos estatales y asociaciones sociales y civiles que empiezan a adoptar e implementar los programas educativos y formativos, al reconocer su importante valor como agentes de reinserción social y laboral de menores infractores, dadas sus innumerables contribuciones para la facilitación y el fortalecimiento de los principales mecanismos de cohesión social y laboral, así como para atenuar y corregir, de manera considerable, las principales desigualdades sociales y laborales, con lo que garantizan la creación de las condiciones propiciatorias para luchar contra la exclusión social y laboral de los colectivos sociales más deprimidos y desfavorecidos (Robert, Weinberg y Gould, 2010).

Por tanto, y como principal conclusión del estudio, es posible aseverar que las actividades educativas y formativas, siempre y cuando se desarrollen con cierta continuidad en el tiempo y se participe de las mismas de forma adecuada y responsable, pueden llegar a revelarse como un destacado instrumento didáctico y metodológico, no sólo para desarrollar y mejorar diversos aspectos cognitivos e intelectuales en los individuos, sino también para consolidar en sus usuarios habituales una serie de valores éticos y morales (empatía, solidaridad, tolerancia, cooperación, sacrificio, autoestima, autocontrol, libertad, paz, entre otros) que les va a facilitar, de manera significativa, el desarrollo y la gestión de su proceso de integración social, comunitaria y laboral, así como el establecimiento de relaciones e interacciones sociales y laborales mucho más ricas, sólidas y eficientes (Fernández-Campoy, Aguilar-Parra, Lorenzo-Torrecillas y Manzano-León, 2016).

Junto a las excelencias y potencialidades de los programas educativos y formativos como elementos generadores de valores y de conductas ampliamente reconocidas y aceptadas por las principales estructuras de los entramados sociales, comunitarios y laborales, que, sin duda, van a faci- 
litar a sus usuarios una rápida y efectiva integración social y laboral, también es posible centrarse en su destacada importancia como elementos limitantes o inhibidores ante el desarrollo de diversos valores y pautas conductuales (violencia, manipulación, consumismo, hedonismo, triunfalismo, utilitarismo, entre otros) que, al experimentar altos niveles de marginalidad y de desprestigio social y laboral, pueden acabar obstaculizando los potenciales niveles de desarrollo y de integración social, comunitaria y laboral de los sujetos que los acaben llevando a la práctica (Pardo, 2008).

En última instancia, y a la luz de los diversos datos y argumentaciones empíricas que se han ido analizando y desgranando como consecuencia del desarrollo de la presente investigación científica, parece necesario concluirla determinando que los programas educativos y formativos, además de revelarse como un extraordinario recurso didáctico y metodológico que posibilita el desarrollo de valores ampliamente reconocidos dentro de los contextos sociales, comunitarios y laborales, también consiguen limitar la aparición de otro tipo de valores que tienen una negativa prensa social, comunitaria y laboral, con lo que acaban conformando un escenario propicio para la configuración de diversos aspectos cognitivos, intelectuales y actitudinales que terminarán resultando capitales, en el sentido de que van a conseguir proporcionar a sus usuarios habituales los recursos metodológicos y procedimentales necesarios para que lleguen a convertirse, en un futuro no muy lejano, en ciudadanos activos y de pleno derecho que logren contribuir al crecimiento, a la transformación y a la mejora de las principales estructuras sociales, comunitarias y laborales (Fernández-Campoy, Aguilar-Parra, Trigueros, Manzano-León y Alcaraz-Ibáñez, 2015).

\section{REFERENCIAS.}

Alpízar, D., Calvo, S., Garita, M., Méndez, M., Mora, A., Loíra, D. y Varela, T. (2010). Importancia de inculcar valores en menores de edad como prevención de la violencia. Revista de Medicina Legal, 28(1), 31-37.

Babiss, L. A. y Gangwisch, J. E. (2010). Sports Participation as a Protective Factor Against Depression and Suicidal Ideation in Adolescents as Mediated by Self-Esteem and Social Support. Global Congress of Maternal and Infant Health, celebrado en Barcelona los días 22, 23, 24, 25 y 26 de Septiembre de 2010.

BLATIER, C. (2002). La délinquance des mineurs. L enfant, le psychologue, le droit. Grenoble: Editorial Presses Universitaires de Grenoble.

Buendía, L. (1999). Modelos de análisis de la investigación educativa. Sevilla: Alfar.

BuRt, M., Resnick, G. y Novick, E. (1998). Building Supportive Communities for at risk adolescents. Washington: American Psychological Association Press.

CAstaño, M. E. (2006). Otra forma de resolver conflictos. El busgosu, 5(2), 54-57.

Coy, E. y TORRente, G. (1997). Intervención con menores infractores: su evolución en España. Anales de Psicología, 13(1), 39-49.

Debarbieux, E. y Blaya, C. (2006). Clima y violencia escolar: un estudio comparativo entre España y Francia. Revista de Educación, 339(3), 293-315.

De la Rosa, J. M. (2003). El fenómeno de la delincuencia juvenil: Causas y tratamientos. Encuentros multidisciplinares, 5(13), 21-35.

Estévez, E., Martínez, B., Moreno, D. y Musitu, G. (2006). Relaciones familiares, rechazo entre iguales y violencia escolar. Cultura y Educación, 3(1), 335-344.

FarRington, D. (2000). Explaining and Preventing Crime. Criminology, 38(1), 12-19.

FERnÁndez-CAmpoy, J. M. (2008). La integración de sujetos inadaptados socialmente. Un estudio de caso: El centro de menores "Tierras de Oria". Granada: Editorial de la Universidad de Granada.

FERnÁndez-CAmpoY, J. M. (2014). Trastornos conductuales en adolescentes infractores. I 


\section{LA RELEVANCIA DE LOS PROGRAMAS EDUCATIVOS Y FORMATIVOS COMO ELEMENTOS FAVORECEDORES DEL PROCESO DE REINSERCIÓN SOCIAL Y LABORAL DE LOS MENORES INFRACTORES}

Congreso Internacional de Necesidades Específicas de Apoyo Educativo, celebrado en Almería los días 26, 27 y 28 de Noviembre de 2014.

Fernández-Campoy, J. M., Aguilar-Parra, J. M. y Alías-García, A. (2012). Influencia de la actividad física y deportiva en la salud y autoestima de las personas. VII Congreso Internacional de la Asociación Española de Ciencias del Deporte, celebrado en Granada los días 15, 16 y 17 de Noviembre de 2012.

Fernández-Campoy, J. M., Aguilar-Parra, J. M. y Álvarez, J. (2013). La formación académica y profesional de los menores infractores del centro de menores "Tierras de Oria". Revista de Educación, 360(1), 211-242.

Fernández-Campoy, J. M., Aguilar-Parra, J. M. y López-LiRia, R. (2015). El perfil del menor infractor desde la teoría científica. III Congreso Internacional sobre Intervención Social y Educativa en Grupos Vulnerables, celebrado en Almería los días 25, 26 y 27 de Noviembre de 2015.

Fernández-Campoy, J. M., Aguilar-Parra, J. M., Álvarez, J., Pérez-Gallardo, E. R. y Salguero, D. (2013). Violencia escolar: rasgos definitorios y prácticas encaminadas a la prevención del fenómeno. International Journal of Developmental and Educational Psychology, 2(1), 131-138.

Fernández-Campoy, J. M., Aguilar-Parra, J. M., Lorenzo-Torrecillas, J. J. y Alcaraz-Ibáñez, M. (2016). Analizando las principales señas de identidad de los Procesos de Intervención Socioeducativa que se suelen aplicar a los menores infractores (pp. 415-421). En Pérez-Fuentes, M. C., Gázquez, J. J., Molero, M. M., Martos, A., Simón, M. M. y Barragán, A. B. (Comps.). Variables Psicológicas y Educativas para la intervención en el ámbito escolar (Volumen II). Almería: ASUNIVEP.

Fernández-Campoy, J. M., Aguilar-Parra, J. M., Lorenzo-Torrecillas, J. J. y Manzano-León, A. (2016). Principales programas educativos encaminados a la reinserción social de menores infractores. III Congreso Internacional sobre Diagnóstico y Orientación, celebrado en Jaén los días 8, 9 y 10 de Septiembre de 2016.

Fernández-Campoy, J. M., Aguilar-Parra, J. M., Lorenzo-Torrecillas, J. J. y Salguero, D. (2016). El valor de los programas deportivos y de actividad física como agentes de reinserción social de los menores infractores (pp. 72-83). En Gallego, J., Alcaraz-Ibáñez, M., Aguilar-Parra, J. M., Cangas, A. J. y Martínez-Luque, D. (Edits.). Avances en actividad física y deportiva inclusiva. Almería: Editorial de la Universidad de Almería.

Fernández-Campoy, J. M., Aguilar-Parra, J. M., Trigueros, R., Manzano-León, A. y Alcaraz-Ibáñez, M. (2015). Prevención de la conducta antisocial a través de la práctica de actividad física y deportiva. En Gallego, J., Alcaraz-Ibáñez, M., Aguilar-Parra, J. M. y Cangas, A. J. (Edits.). IV Congreso Internacional de Deporte Inclusivo. Almería: Editorial de la Universidad de Almería.

Fernández-Campoy, J. M., Pérez-Gallardo, E. R., León, P. y Salguero, D. (2014). Conducta antisocial en infancia y adolescencia: análisis de la problemática y prácticas encaminadas a su prevención. I Congreso Internacional de Necesidades Específicas de Apoyo Educativo, celebrado en Almería los días 26, 27 y 28 de Noviembre de 2014.

GHiso, A. M. (2012). Algunos límites de las respuestas frente a la violencia y la inseguridad en las instituciones educativas. Revista Latinoamericana de Ciencias Sociales, 2(10), 815-824.

Giller, H., Haggel, I. y RutTer, M. (2000). La conducta antisocial de los jóvenes. Cambridge: Universidad de Cambridge.

GonzÁlez, J. y SANTIUSTE, V. (2004). Los conflictos entre los alumnos: características para una convivencia democrática. Revista de Psicología General y Aplicada, 3(1), 361-372.

Howell, J. C. y Hawkins, J. D. (1998). Prevention of Youth Violence. En TonRY, M. y Moore, M. Youth Violence. Chicago: The University of Chicago Press. 
KRMPotic, C. y FarRÉ, M. (2008). Violência social e escola: Um relato empírico na perspectiva de bairros críticos. Revista Katálysis, 2(1), 195-203.

Martínez, B., Musitu, G., Amador, L. V. y Monreal, M. C. (2012). Estatus sociométrico y violencia escolar en adolescentes: implicaciones de la autoestima, la familia y la escuela. Revista Latinoamericana de Psicología, 44(2), 55-66.

Nieto, C. (2010). Las infracciones penales de los jóvenes: una mirada sociológica. Revista Andaluza de Ciencias Sociales, 11(9), 39-51.

Nieto, C. (2012). Perfil de los menores en conflicto de la ley. Trabajo Social y Servicios Sociales, $7(6), 47-60$.

ORTega, R. y Del Rey, R. (2006). La mediación escolar en el marco de la construcción de la convivencia y la prevención de la violencia. Revista de la Asociación de Inspectores de Educación de España, 2(1), 12-25.

PARDo, R. (2008). La transmisión de valores a jóvenes socialmente desfavorecidos a través de la actividad física y el deporte. Estudio múltiple de casos: Getafe, L Alquila y Los Ángeles. Madrid: Editorial de la Universidad Politécnica de Madrid.

Perea, M. B., Calvo, A. L. y Anguiano, A. M. (2010). La familia y la escuela coexistiendo con la violencia escolar. Revista de Trabajo Social y Ciencias Sociales, 58(2), 72-81.

Pérez, V., AmadoR, L. V. y Vargas, M. (2011). Resolución de conflictos en las aulas: un análisis desde la investigación-acción. Revista Interuniversitaria de Pedagogía Social, 18(1), 99-114.

Robert, S., Weinberg, A. y Gould, D. (2010). Fundamentos de psicología del deporte y del ejercicio físico. Madrid: Panamericana.

SCHONERT-REICHL, P. (2000). Children and youth at risk: some conceptual considerations. Canadá: Pan Canadian Education Research Agenda Symposium.

SHaw, M. (2001). Invirtiendo en los jóvenes 12-18 años: enfoques internacionales para prevenir el crimen y la victimización. Montreal: Centro Internacional para la Prevención del Crimen.

Sherman, L. et Al. (1997). Preventing Crime: What works, what doesn t, what promising. Washington: National Institute of Justice. 
